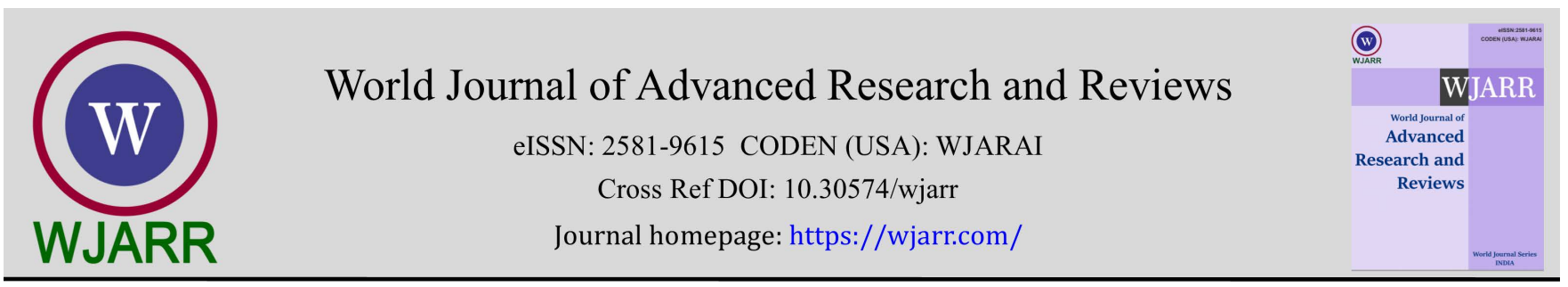

(RESEARCH ARTiClE)

\title{
Nutritional and health benefit knowledge of milk and dairy products consumption among medical students at Benghazi university
}

\author{
Mashallah Mohamed Abdul-Aziz ${ }^{1}$, Aya Abdul-Salam Algomati 1, Timh Salem Alhasi 1, Mailud Saad El-Amari ${ }^{1}$, \\ Abdelmetalab Ferjani Tarhuni ${ }^{2}$, Abdullah Ramadan Sheikhi ${ }^{3}$ and Ali Ateia Elmabsout $1{ }^{*}{ }^{*}$ \\ ${ }^{1}$ Department of nutrition, faculty of public health, university of Benghazi, Benghazi, Libya. \\ 2 Department of Environment health, Faculty of public health, University of Benghazi, Benghazi, Libya. \\ ${ }^{3}$ Curtin university, Perth, Western Australia, Australia.
}

World Journal of Advanced Research and Reviews, 2021, 12(01), 162-174

Publication history: Received on 28 August 2021; revised on 06 October 2021; accepted on 08 October 2021

Article DOI: https://doi.org/10.30574/wjarr.2021.12.1.0499

\begin{abstract}
Backgrounds: Milk well- known important source of human nutrition and contain considerable amounts of macro and micronutrients and have enormous health benefit. This study was performed to determine the nutritional knowledge and health benefit knowledge of diary and dairy products consumption among medical students at Benghazi university.

Methodology: This a cross section study comprised of 541 students from branches of medical faculties in which 290 female and 251 male. Data were collected by using a semi-constructed questionnaire. Data was analysis by either mean \pm SD or frequencies and percentages according to the natural of data. Chi-square test was used at $\alpha<0.05$.

Results: The result of present work revealed that milk was consumed by one serving a day (53\%) $(P<0.05)$, and among types of milks, whole fat milk significantly consumed $(P<0.05)$. Our result also shown that dairy products were highly consumed by one serving/day. Furthermore, $65.2 \%$ of the students drink milk with tea $(P=0.000)$. Nutrition knowledge of milk and dairy products reported that students have good knowledge score for nutritional knowledge questions and poor knowledge scores for questions related to health benefit with exception question on oral health $(P<0.05)$. Even though, the nutritional status of the students reveals that they fall under normal weight categories. However there were abnormal low levels of $\mathrm{Hb}$, vitamin $\mathrm{D}$ have been found and abnormal high levels of calcium among male students.
\end{abstract}

Conclusion Dairy consumption among students was relatively higher with good nutritional knowledge of dairy and dairy products consumptions and poor knowledge regarding health benefit. The result of this study suggested that nutritional education program might be in great importance of dairy and dairy product knowledge and benefit. The data of this study needed to be validated in large samples.

Keywords: Dairy consumption; Milk, Students; Nutrition status; Knowledge; Health benefit

\section{Introduction}

Milk is very- known source of human nutrition since 4000 BC $[1,2]$. Milk consumption can increase intake of not only calcium but also overall nutrient and milk contains all the different nutrients that humans need as well $[3,4]$.

A little knowledge provided in the literature in which few people know that milk is a source of protein, full of B-group vitamins (thiamin, riboflavin, niacin, vitamin B6, B12 and folate), vitamin A, vitamin C, magnesium, and zinc [5]. Seventy

\footnotetext{
${ }^{*}$ Corresponding author: Ali Ateia Elmabsout

Department of nutrition, faculty of public health, university of Benghazi, Benghazi, Libya.

Copyright $(2021$ Author(s) retain the copyright of this article. This article is published under the terms of the Creative Commons Attribution Liscense 4.0.
} 
percent of calcium and $16 \%$ of potassium in the diet are derived from dairy and dairy products [6]. Furthermore, milk also provide in about $30 \%$ of phosphorus, $14 \%$ of magnesium, $15 \%$ of zinc, $18 \%$ of protein, $16 \%$ of vitamin A, $18 \%$ of vitamin B12, and $25 \%$ of riboflavin [5]. In addition, Milk has a good source of carbohydrates lactose, and small quantities of the fatty acids and the main fatty acid present in the milk is monounsaturated [6].

Milk is staple in human consumption, contributing calcium intake which is an important for builds strong bones, proteins for the brains function and muscles to develop and for normal growth as well [7]. Lack of milk in the diet can participate in calcium and vitamin D deficiencies and poor health [8]. Therefore, many dietary guidelines stress milk and dairy foods as an essential component of a healthy diet for all people, regardless of their age [9].

A number of studies have shown that milk and dairy products contribute to bone health and help to prevent cardiovascular diseases, high blood pressure, and Type 2 diabetes [10,11]. Studies also found that that frequent consumption of dairy foods and milk should be recommended in order to prevent periodontal disease [12, 13]. Moreover, Calcium derived from milk intake has beneficial in reducing cholesterol absorption, and in controlling body weight and blood pressure [14]. However, recent conflict work has emerged about the benefits compared with harms of dairy fat, including concerns over long-term effects [15]. Some traditional diet-heart study held that consumption of fat, and particularly saturated fat associated with arise levels of total and low-density lipoprotein (LDL) cholesterol which result in developing of coronary heart disease [16]. Based on this knowledge, dietary guidelines in some countries and international authorities recommend consumption of low-fat dairy foods [17]. On the other hands, total dairy product intake has not been found associated with increased overall cancer mortality risk [18].

Beside of nutritional values of milk and dairy products there is biologically active compounds (bioactive peptides, probiotic bacteria, antioxidants, vitamins, specific proteins, oligosaccharides, organic acids, highly absorbable calcium, conjugated linoleic acid and others) have important for human functioning and health [19].

Milk is very important during growth periods, especially when growth occurs rapidly, for instance, in adolescence [20]. Since, young adults continue to grow in early adulthood, accurate knowledge and health benefit about milk will lead them to increase their milk consumption. Therefore a according to our knowledge little or no study conducts at medical students for milk nutritional and health point of view and medical student supposed to have more awareness regarding knowledge and benefit of milk and dairy products consumption. For the students, milk and dairy products overall provide better or worse health, and increase or lowering risk of some diseases and all-cause of mortality. Hence, the aim of the present work was to study the nutritional and health benefit knowledge of dairy and dairy products consumption among medical students.

\section{Material and methods}

\subsection{Subjects}

A cross sectional study was conducted from end of 2019 to the $15^{\text {th }}$ of March 2020 on 541 students from medical faculties branches by which 290 female and 252 male. All participants with age between 18-26 years old were selected for the study. Medical faculties in Benghazi university consist of faculty of medicine, dental, pharmacy, medical biotechnology and public health faculty. All the subjects selected for the study were fully informed of the purpose and procedure of the investigation and provided consent at the outset.

\subsection{Questionnaire Development}

A self-administered designed questionnaire including demographic data, anthropometric data and questions for knowledge, and health benefit of milk and dairy consumption. The questionnaire contains in total twenty five questions. The questions were divided between $4-6$ sections including: consumption pattern, knowledge and health benefit of the students about milk consumption.

\subsection{Procedure}

Questionnaires were distributed to the randomly selected students. The details study procedures were explain to the students and to indicate their rights as students. The questionnaire including questions including demographic profile, consumption pattern, nutritional knowledge of the students. After completing questionnaires, the students were asked for measuring their weight and height. Modified food frequency questionnaire was used and focusing on milk and dairy food intake. 


\subsection{Anthropometric measurements}

Weight and height were measured as previously described [21] by which weight was measured with minimal cloths and nearest to $0.1 \mathrm{~kg}$ and height measured with bare foot by measuring tape nearest to $0.2 \mathrm{~cm}$. BMI was calculated as prescribed by $\mathrm{WHO}, \mathrm{BMI}=$ weight $\mathrm{kg} /$ height $\mathrm{m}^{2}$. BMI categorized as following:

$\mathrm{BMI}<18.5$ underweight

BMI $>18.5$ and $<24.9$ normal weight

BMI 25 and $<29.9$ over weight

BMI 30 and above obese.

\subsection{Nutritional and health benefit knowledge of milk consumption questions}

For scoring nutritional knowledge and health benefit considered that if the answer of the question less than $50 \%$ is define as poor knowledge and if $50 \%$ or more is defined as good knowledge.

\subsection{Ethics}

All of the procedures involving human subjects were approved by local Medical Ethics committee. Written consent was obtained from the students before the study began.

\subsection{Statistical Analysis}

IBM SPSS (predictive analytics software and solutions) version 22.0 (International Business Machines Corporation, Armonk, NY, USA) was used for analysis. Values were presented as mean \pm SD for quantitative data or frequencies and or percentage for qualitative data. Chi-squared analysis was used to compare socio-demographic, knowledge and health benefit of milk and dairy products consumption characteristics among participants with different knowledge. A P value less than 0.05 was considered as statistically significantly different.

\section{Results}

The mean and standard deviation (SD) of participants' ages were 23.5 \pm 3 . Participants' age ranged from 18 to 26 years old. The highest and significant age of the students participate in the study were those between 21-23 years old (50.5\%) $P=0.000$, followed by age groups $24-26$ years old (31\%) (Table 1). Furthermore, more than $50 \%$ of the students were female $(53.6 \%)$ and male represent about (46.4\%) the gender (Figure 1). In regard family income of the students, more than 50\% have an average income between 500-1000 LD, about one fourth have an average income more than $1000 \mathrm{LD}$ (Figure 2).

Table 1 Age distribution of the students

\begin{tabular}{|c|c|c|c|c|}
\hline & & $\mathbf{N}$ & N \% & $P$ values \\
\hline \multirow{4}{*}{ Ages } & $18-20$ & 101 & $18.7 \%$ & \\
\hline & $21-23$ & 273 & $50.5 \%$ & 0.000 \\
\hline & $24-26$ & 167 & $30.9 \%$ & \\
\hline & Total & 541 & $100.0 \%$ & \\
\hline
\end{tabular}

541 participants were recruited from branches of medical faculties by which 153 (28.3\%) from medicine, 165 (30.5\%) from pharmacy, 149 (27.5\%) from public health, 44 (8.1\%) from dental, and 30 (5.5\%) from medical technology, in which students class ranking as the following: about $30 \%$ of the students belong to third class followed by $26.4 \%$ from second and first and fourth class $17.9 \%$ and 18.7\% respectively whereas the fifth class being the lowest (7.8\%) (Table 2). 


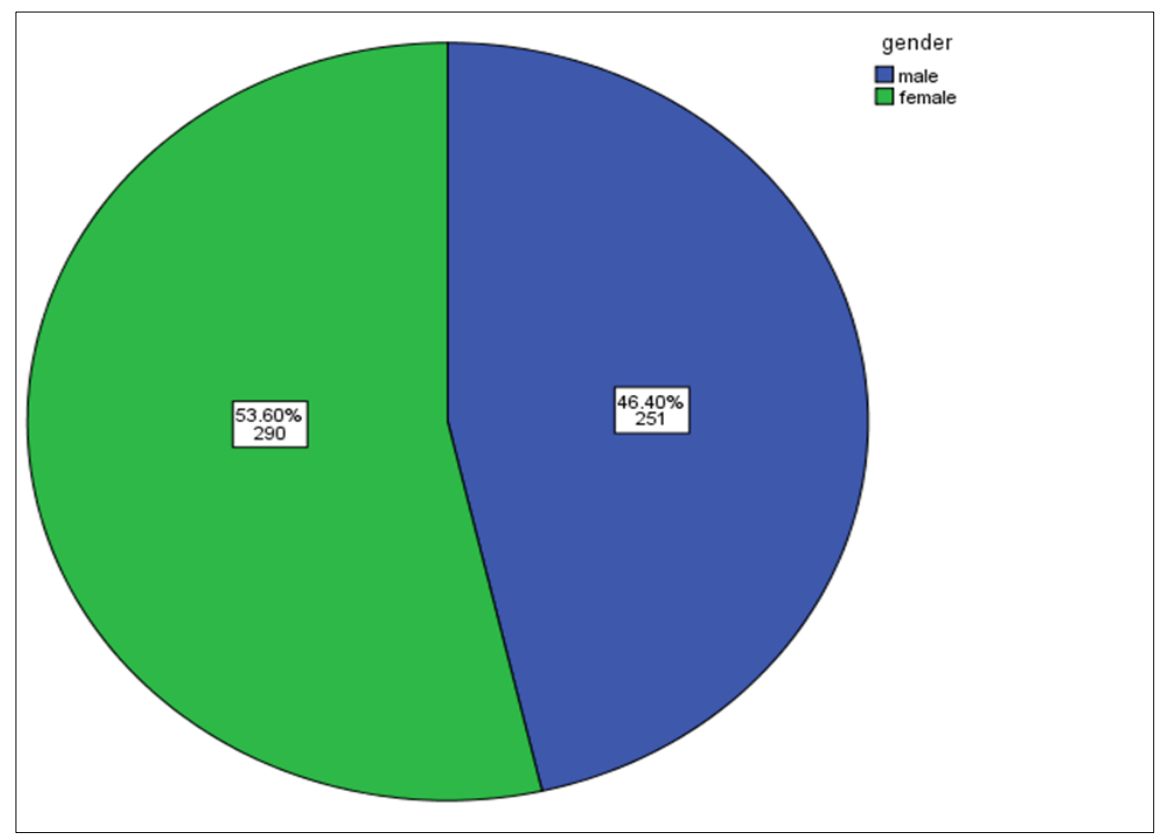

Figure 1 Gender distribution

Table 2 Students from different branches and classes of medical faculties

\begin{tabular}{|c|l|c|c|}
\hline \multicolumn{2}{|c|}{} & N & N \% \\
\hline \multirow{5}{*}{ Faculties } & public health & 149 & $27.5 \%$ \\
\cline { 2 - 4 } & pharmacy & 165 & $30.5 \%$ \\
\cline { 2 - 4 } & dental & 44 & $8.1 \%$ \\
\cline { 2 - 4 } & medicine & 153 & $28.3 \%$ \\
\cline { 2 - 4 } & medical technology & 30 & $5.5 \%$ \\
\cline { 2 - 4 } & Total & 541 & $100.0 \%$ \\
\hline \multirow{5}{*}{ Class } & first & 97 & $17.9 \%$ \\
\cline { 2 - 4 } & second & 143 & $26.4 \%$ \\
\cline { 2 - 4 } & third & 158 & $29.2 \%$ \\
\cline { 2 - 4 } & fourth & 101 & $18.7 \%$ \\
\cline { 2 - 4 } & fifth & 42 & $7.8 \%$ \\
\cline { 2 - 4 } & Total & 541 & $100.0 \%$ \\
\hline
\end{tabular}

The milk drinking habits of the students were shown in Table 3. Table 3 shown that $82.6 \%$ of the students were stated consumed milk. Milk is mostly consumed by one cup a day (53\%) and two cups were come in the next with around $21.6 \%$ and three cups a day being the least (8.1\%). The questions regarding types of milk consumption reported that, whole milk was highly significant reported $(60.1 \%) P=0.000$. Pasteurized, raw and low fat reported with lowest percentages $30 \%, 7.8 \%$ and $17 \%$ respectively. 
Table 3 Milk habits and types of milk consumption

\begin{tabular}{|c|c|c|c|c|}
\hline & & $\mathbf{N}$ & N \% & $P$ values \\
\hline \multirow{3}{*}{ Drink milk } & yes & 447 & $82.6 \%$ & \\
\hline & no & 94 & $17.4 \%$ & \\
\hline & Total & 541 & $100.0 \%$ & \\
\hline \multirow{5}{*}{ Cups per day } & No drink & 94 & $17.4 \%$ & \\
\hline & one cup & 286 & $52.9 \%$ & 0.000 \\
\hline & two cups & 117 & $21.6 \%$ & \\
\hline & three cups and more & 44 & $8.1 \%$ & \\
\hline & Total & 541 & $100.0 \%$ & \\
\hline \multirow{3}{*}{ Pasteurized milk } & yes & 162 & $29.9 \%$ & \\
\hline & no & 379 & $70.1 \%$ & \\
\hline & Total & 541 & $100.0 \%$ & \\
\hline \multirow{3}{*}{ Raw milk } & yes & 42 & $7.8 \%$ & \\
\hline & no & 499 & $92.2 \%$ & \\
\hline & Total & 541 & $100.0 \%$ & \\
\hline \multirow{3}{*}{ Low fat milk } & yes & 92 & $17.0 \%$ & \\
\hline & no & 449 & $83.0 \%$ & \\
\hline & Total & 541 & $100.0 \%$ & \\
\hline \multirow{3}{*}{ Whole milk } & yes & 325 & $60.1 \%$ & 0.000 \\
\hline & no & 216 & $39.9 \%$ & \\
\hline & Total & 541 & $100.0 \%$ & \\
\hline
\end{tabular}

Chi-square test was performed and considered significant at $\alpha<0.05$

Analysis of food frequency of study sample regarding the amount and types of dairy products revealed the following results. There was an increase in the consumption of dairy products (97.2\%) include $91.1 \%$ consumed cheese, $73.8 \%$ yogurt, and 54.9\% cream. These dairy products were found most often consumed once/day (57.5\%) and 36.4\% twice/day. When milk consumption is examined; $65.2 \%$ of the students drink milk with tea $(P=0.000)$, and least drink it with coffee (15.2\%) and moderately drink it alone (20\%) (Table 4).

The answers of the question about nutrition knowledge of the milk were shown in Table 5. Among the six questions, over half of the students (59.1\%) have answer milk contain lacto-globulin, approximately $92.2 \%, 90.9 \%, 98 \%$ and $84.8 \%$ have good knowledge of that milk rich sources of protein, lactose, calcium and good sources of energy, more than two third were reported that milk containing vitamin D (Table 5).

In response to questions of health benefit of diary and dairy products, poor knowledge have been reported in which about 36.6\%, 29.4\% , 14.2\% , 27.2\% and 33.1\% answered "Yes" for dairy and dairy products prevent CVD, colorectal cancer, ovarian cancer, lose weight, and reduce risk of T2DM respectively Moderate knowledge have been reported by the student for the questions milk protect oral health (58.8\%) (Table 6).

Further investigation of health risk and dairy product consumption shown in table 7. Poor knowledge reported in the table 7 in which 19\%, 47\% and 35.7\% answered "Yes" for excessive consumption of skim milk cause acne, Cheese contain large amount of sodium, and Pasteurized cheese cause constipation respectively. 
Table 4 Dairy products consumption

\begin{tabular}{|c|l|c|c|c|}
\hline \multicolumn{2}{|c|}{} & $\mathbf{N}$ & $\mathbf{N} \%$ & P values \\
\hline \multirow{4}{*}{ Dairy product } & yes & 526 & $97.2 \%$ & \\
\cline { 2 - 5 } & no & 15 & $2.8 \%$ & \\
\cline { 2 - 5 } & Total & 541 & $100.0 \%$ & \\
\hline \multirow{4}{*}{ cheese } & yes & 493 & $91.1 \%$ & \\
\cline { 2 - 5 } & no & 48 & $8.9 \%$ & \\
\cline { 2 - 5 } & Total & 541 & $100.0 \%$ & \\
\hline \multirow{5}{*}{ cream } & yes & 399 & $73.8 \%$ & \\
\cline { 2 - 5 } & no & 142 & $26.2 \%$ & \\
\cline { 2 - 5 } & Total & 541 & $100.0 \%$ & \\
\hline \multirow{5}{*}{ How often } & yes & 297 & $54.9 \%$ & \\
\cline { 2 - 5 } & no & 244 & $45.1 \%$ & \\
\cline { 2 - 5 } & Total & 541 & $100.0 \%$ & \\
\hline & once & 311 & $57.5 \%$ & 0.000 \\
\cline { 2 - 5 } & twice & 197 & $36.4 \%$ & \\
\cline { 2 - 5 } & three times & 33 & $6.1 \%$ & \\
\cline { 2 - 5 } & Total & 541 & $100.0 \%$ & \\
\hline \multirow{5}{*}{ Habit of drink milk } & coffee & 82 & $15.2 \%$ & \\
\cline { 2 - 5 } & tea & 353 & $65.2 \%$ & 0.000 \\
\cline { 2 - 5 } & milk only & 106 & $19.6 \%$ & \\
\cline { 2 - 5 } & Total & 541 & $100.0 \%$ & \\
\hline
\end{tabular}

Chi-square test was performed and considered significant at $\alpha<0.05$

Table 5 Milk knowledge questions

\begin{tabular}{|l|l|c|c|c|}
\hline \multicolumn{2}{|c|}{} & \multicolumn{1}{|c|}{$\mathbf{N}$} & $\mathbf{N} \%$ & P values \\
\hline \multirow{4}{*}{ Lactoglobulin } & yes & 320 & $59.1 \%$ & 0.00 \\
\cline { 2 - 5 } & no & 221 & $40.9 \%$ & \\
\cline { 2 - 5 } & Total & 541 & $100.0 \%$ & \\
\hline \multirow{4}{*}{ Protein } & yes & 499 & $92.2 \%$ & 0.000 \\
\cline { 2 - 5 } & no & 42 & $7.8 \%$ & \\
\cline { 2 - 5 } & Total & 541 & $100.0 \%$ & \\
\hline \multirow{5}{*}{ Lactose } & yes & 492 & $90.9 \%$ & 0.000 \\
\cline { 2 - 5 } & no & 49 & $9.1 \%$ & \\
\cline { 2 - 5 } & Total & 541 & $100.0 \%$ & \\
\hline Calcium & yes & 530 & $98.0 \%$ & 0.000 \\
\cline { 2 - 5 } & no & 11 & $2.0 \%$ & \\
\cline { 2 - 5 } & Total & 541 & $100.0 \%$ & \\
\hline Vitamin D & yes & 406 & $75.0 \%$ & 0.000 \\
\cline { 2 - 5 } & no & 135 & $25.0 \%$ & \\
\cline { 2 - 5 } & Total & 541 & $100.0 \%$ & \\
& yes & 459 & $84.8 \%$ & 0.000 \\
& no & 82 & $15.2 \%$ & \\
& Total & 541 & $100 \%$ & \\
\hline
\end{tabular}

Chi-square test was performed and considered significant at $\alpha<0.05$ 
Table 6 Health benefit of dairy and dairy products consumption equestions

\begin{tabular}{|c|c|c|c|c|}
\hline & & $\mathbf{N}$ & N\% & $P$ values \\
\hline \multirow[t]{3}{*}{ Prevent CVD } & yes & 198 & $36.6 \%$ & \\
\hline & no & 343 & $63.4 \%$ & \\
\hline & Total & 541 & $100.0 \%$ & \\
\hline \multirow[t]{3}{*}{ Prevent colorectal cancer } & yes & 159 & $29.4 \%$ & \\
\hline & no & 382 & $70.6 \%$ & \\
\hline & Total & 541 & $100.0 \%$ & \\
\hline \multirow[t]{3}{*}{ Prevent ovarian cancer } & yes & 77 & $14.2 \%$ & \\
\hline & no & 464 & $85.8 \%$ & \\
\hline & Total & 541 & $100.0 \%$ & \\
\hline \multirow[t]{3}{*}{ Lose weight } & yes & 147 & $27.2 \%$ & \\
\hline & no & 394 & $72.8 \%$ & \\
\hline & Total & 541 & $100.0 \%$ & \\
\hline \multirow[t]{3}{*}{ Reduces risk T2DM } & yes & 179 & $33.1 \%$ & \\
\hline & no & 362 & $66.9 \%$ & \\
\hline & Total & 541 & $100.0 \%$ & \\
\hline \multirow[t]{3}{*}{ Oral health } & yes & 318 & $58.8 \%$ & 0.000 \\
\hline & no & 223 & $41.2 \%$ & \\
\hline & Total & 541 & $100.0 \%$ & \\
\hline
\end{tabular}

Chi-square test was performed and considered significant at $\alpha<0.05$

Table 7 Health risk of dairy products consumptions

\begin{tabular}{|l|c|c|c|}
\hline \multirow{2}{*}{ Excessive consumption of skim milk cause acne } & yes & 103 & $19.0 \%$ \\
\cline { 2 - 4 } & no & 438 & $81.0 \%$ \\
\cline { 2 - 4 } & Total & 541 & $100.0 \%$ \\
\hline Cheese contain large amount of sodium & yes & 254 & $47.0 \%$ \\
\cline { 2 - 4 } & no & 287 & $53.0 \%$ \\
\cline { 2 - 4 } & Total & 541 & $100.0 \%$ \\
\hline \multirow{2}{*}{ Pasteurized cheese cause constipation } & yes & 193 & $35.7 \%$ \\
\cline { 2 - 4 } & no & 348 & $64.3 \%$ \\
\cline { 2 - 4 } & Total & 541 & $100.0 \%$ \\
\hline
\end{tabular}

In Table 8, nutritional status of students was evaluated by using BMI (body mass index). The results of the table 8 showed that most of the study participants fall in the normal weight (58.6\%) On the other hand, there was also a decrease in the participants having obesity and underweight $7.9 \%$ and $10.9 \%$ respectively. The mean weight $66.0 \pm 5 \mathrm{~kg}$. The mean Body Mass Index (BMI) of the study participants was $23.64 \pm 4.0 \mathrm{~kg} / \mathrm{m}^{2}$ indicating that majority of the study participants had normal weight. 
The nutritional lab indices shown that, both male and female have lowered $\mathrm{Hb}$ and vitamin $\mathrm{D}$ levels. However serum calcium shown great differences in which normal values for female and abnormal high values for male (Table 9).

Table 8 Anthropometric measurements

\begin{tabular}{|c|c|c|c|c|}
\hline & & Mean \pm SD & $\mathbf{N}$ & N \% \\
\hline \multicolumn{2}{|l|}{ weight } & $66 \pm 5$ & & \\
\hline \multicolumn{2}{|l|}{ BMI } & $23.64 \pm 4$ & & \\
\hline \multirow[t]{5}{*}{ BMI categories } & Underweight & & 59 & $10.9 \%$ \\
\hline & Normal & & 317 & $58.6 \%$ \\
\hline & Overweight & & 124 & $22.9 \%$ \\
\hline & Obese & & 41 & $7.6 \%$ \\
\hline & Total & & 541 & $100.0 \%$ \\
\hline
\end{tabular}

Table 9 Lab values for some nutritional indices

\begin{tabular}{|l|c|c|}
\hline \multirow{2}{*}{} & \multicolumn{2}{|c|}{ gender } \\
\cline { 2 - 3 } & male & female \\
\cline { 2 - 3 } & Mean \pm SD & Mean \pm SD \\
\hline $\mathrm{Hb}$ & $12.87 \pm 5$ & $10.69 \pm 3$ \\
\hline $\mathrm{Ca}^{++}$ & $12.87 \pm 7$ & $9.17 \pm 4$ \\
\hline $\mathrm{Vit} . \mathrm{D}$ & $16.20 \pm 8$ & $14.73 \pm 6$ \\
\hline
\end{tabular}

Table 10 Relationship between health benefit of dairy product consumption and students' knowledge

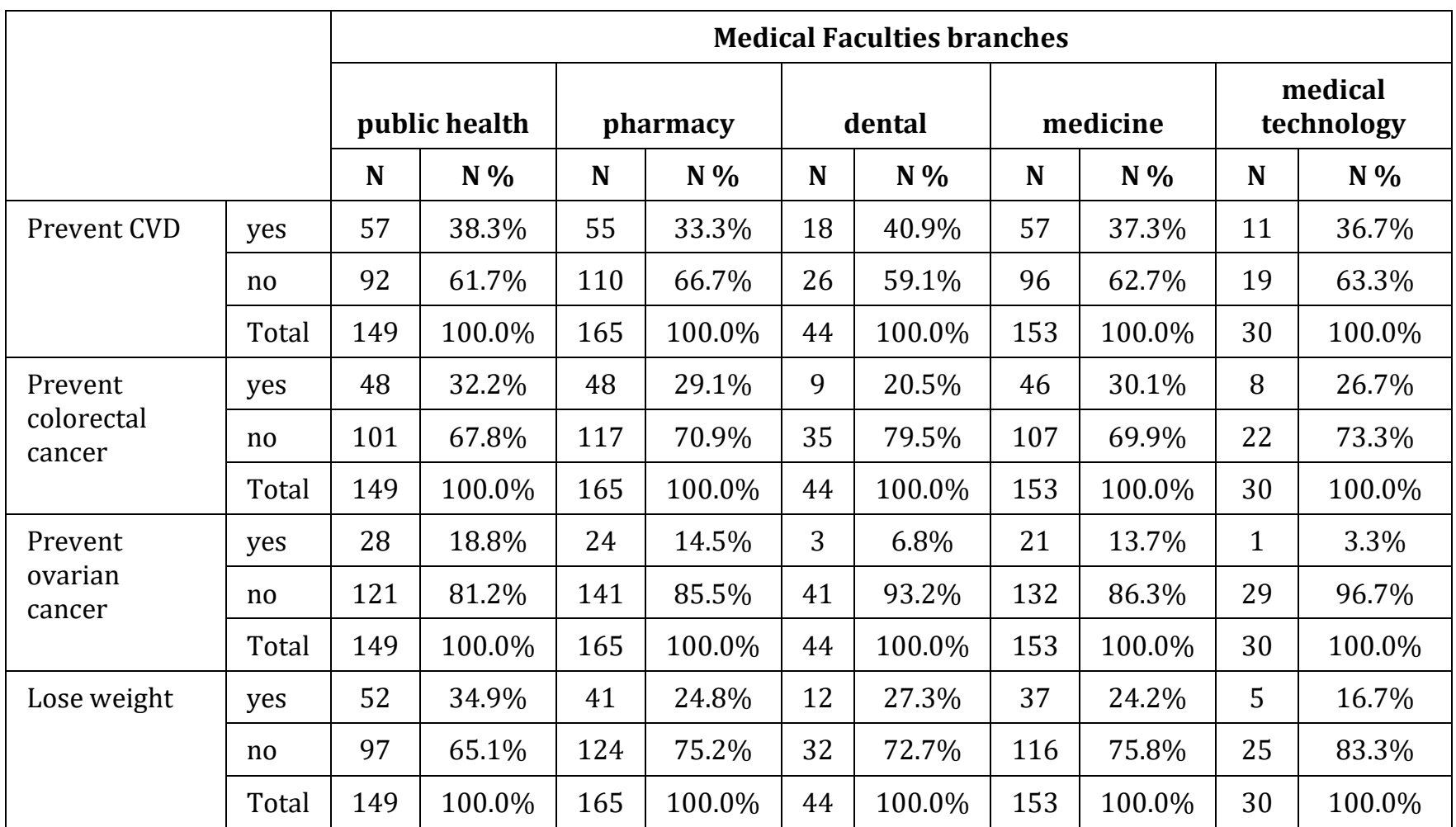




\begin{tabular}{|l|l|c|c|c|c|c|c|c|c|c|c|}
\hline \multirow{2}{*}{$\begin{array}{l}\text { Reduces } \\
\text { DM }\end{array}$} & yes & 57 & $38.3 \%$ & 49 & $29.7 \%$ & 13 & $29.5 \%$ & 50 & $32.7 \%$ & 10 & $33.3 \%$ \\
\cline { 2 - 11 } & no & 92 & $61.7 \%$ & 116 & $70.3 \%$ & 31 & $70.5 \%$ & 103 & $67.3 \%$ & 20 & $66.7 \%$ \\
\cline { 2 - 11 } & Total & 149 & $100.0 \%$ & 165 & $100.0 \%$ & 44 & $100.0 \%$ & 153 & $100.0 \%$ & 30 & $100.0 \%$ \\
\hline \multirow{3}{*}{\begin{tabular}{l} 
Oral health \\
\cline { 2 - 11 }
\end{tabular}} & yes & 92 & $61.7 \%$ & 88 & $53.3 \%$ & 27 & $61.4 \%$ & 94 & $61.4 \%$ & 17 & $56.7 \%$ \\
\cline { 2 - 11 } & no & 57 & $38.3 \%$ & 77 & $46.7 \%$ & 17 & $38.6 \%$ & 59 & $38.6 \%$ & 13 & $43.3 \%$ \\
\cline { 2 - 11 } & Total & 149 & $100.0 \%$ & 165 & $100.0 \%$ & 44 & $100.0 \%$ & 153 & $100.0 \%$ & 30 & $100.0 \%$ \\
\hline
\end{tabular}

Chi-square test was performed and considered significant at $\alpha<0.05$

Dairy and dairy products knowledge was evaluated among medical faculties branches as shown in table 10. Public health and medicine faculty students have good knowledge for oral health $P=0.004$ while the other faculties lack such knowledge (Table 10). For the knowledge for other disease listed in table 10 did not reveal any differences.

The knowledge for milk containing lactoglobulin was high significant among students from public health, pharmacy and medicine faculty $(\mathrm{P}=0.000)$ (Table 11) whereas similar trend for all milk components and medical faculties branches (Table 11).

Table 11 Nutritional knowledge of milk composition of student of medical faculties

\begin{tabular}{|c|c|c|c|c|c|c|c|c|c|c|c|}
\hline & & \multicolumn{10}{|c|}{ Medical Faculties branches } \\
\hline & & \multicolumn{2}{|c|}{ public health } & \multicolumn{2}{|c|}{ pharmacy } & \multicolumn{2}{|c|}{ dental } & \multicolumn{2}{|c|}{ medicine } & \multicolumn{2}{|c|}{$\begin{array}{l}\text { medical } \\
\text { technology }\end{array}$} \\
\hline & & $\mathbf{N}$ & N \% & $\mathbf{N}$ & N \% & $\mathbf{N}$ & $\mathrm{N} \%$ & $\mathbf{N}$ & $\mathrm{N} \%$ & $\mathbf{N}$ & $\mathrm{N} \%$ \\
\hline \multirow[t]{3}{*}{ protein } & yes & 133 & $89.3 \%$ & 160 & $97.0 \%$ & 41 & $93.2 \%$ & 138 & $90.2 \%$ & 27 & $90.0 \%$ \\
\hline & no & 16 & $10.7 \%$ & 5 & $3.0 \%$ & 3 & $6.8 \%$ & 15 & $9.8 \%$ & 3 & $10.0 \%$ \\
\hline & Total & 149 & $100.0 \%$ & 165 & $100.0 \%$ & 44 & $100.0 \%$ & 153 & $100.0 \%$ & 30 & $100.0 \%$ \\
\hline \multirow[t]{3}{*}{ lactoglobulin } & yes & 94 & $63.1 \%$ & 104 & $63.0 \%$ & 19 & $43.2 \%$ & 94 & $61.4 \%$ & 9 & $30.0 \%$ \\
\hline & no & 55 & $36.9 \%$ & 61 & $37.0 \%$ & 25 & $56.8 \%$ & 59 & $38.6 \%$ & 21 & $70.0 \%$ \\
\hline & Total & 149 & $100.0 \%$ & 165 & $100.0 \%$ & 44 & $100.0 \%$ & 153 & $100.0 \%$ & 30 & $100.0 \%$ \\
\hline \multirow[t]{3}{*}{ lactose } & yes & 138 & $92.6 \%$ & 151 & $91.5 \%$ & 38 & $86.4 \%$ & 140 & $91.5 \%$ & 25 & $83.3 \%$ \\
\hline & no & 11 & $7.4 \%$ & 14 & $8.5 \%$ & 6 & $13.6 \%$ & 13 & $8.5 \%$ & 5 & $16.7 \%$ \\
\hline & Total & 149 & $100.0 \%$ & 165 & $100.0 \%$ & 44 & $100.0 \%$ & 153 & $100.0 \%$ & 30 & $100.0 \%$ \\
\hline \multirow[t]{3}{*}{ calcium } & yes & 145 & $97.3 \%$ & 162 & $98.2 \%$ & 44 & $100.0 \%$ & 149 & $97.4 \%$ & 30 & $100.0 \%$ \\
\hline & no & 4 & $2.7 \%$ & 3 & $1.8 \%$ & 0 & $0.0 \%$ & 4 & $2.6 \%$ & 0 & $0.0 \%$ \\
\hline & Total & 149 & $100.0 \%$ & 165 & $100.0 \%$ & 44 & $100.0 \%$ & 153 & $100.0 \%$ & 30 & $100.0 \%$ \\
\hline \multirow[t]{3}{*}{ Vitamin D } & yes & 111 & $74.5 \%$ & 131 & $79.4 \%$ & 35 & $79.5 \%$ & 109 & $71.2 \%$ & 20 & $66.7 \%$ \\
\hline & no & 38 & $25.5 \%$ & 34 & $20.6 \%$ & 9 & $20.5 \%$ & 44 & $28.8 \%$ & 10 & $33.3 \%$ \\
\hline & Total & 149 & $100.0 \%$ & 165 & $100.0 \%$ & 44 & $100.0 \%$ & 153 & $100.0 \%$ & 30 & $100.0 \%$ \\
\hline
\end{tabular}

The knowledge of the students classes for benefit of milk and dairy products consumptions presented in table 12 . Similar poor knowledge was found in all classes and benefit of dairy and dairy products consumption except for oral health found significant in second, third and fourth classes $(P<0.05)$. (Table 12). 
Table 12 Relationship between student classes and knowledge of health benefit of diary and dairy products consumption

\begin{tabular}{|c|c|c|c|c|c|c|c|c|c|c|c|}
\hline & & \multicolumn{10}{|c|}{ Years (classes) } \\
\hline & & \multicolumn{2}{|c|}{ first } & \multicolumn{2}{|c|}{ second } & \multicolumn{2}{|c|}{ third } & \multicolumn{2}{|c|}{ fourth } & \multicolumn{2}{|c|}{ fifth } \\
\hline & & $\mathbf{N}$ & N \% & $\mathbf{N}$ & N \% & $\mathbf{N}$ & N \% & $\mathbf{N}$ & N \% & $\mathbf{N}$ & N \% \\
\hline \multirow[t]{3}{*}{ 1-Prevent CVD } & yes & 32 & $33.0 \%$ & 47 & $32.9 \%$ & 62 & $39.2 \%$ & 40 & $39.6 \%$ & 17 & $40.5 \%$ \\
\hline & no & 65 & $67.0 \%$ & 96 & $67.1 \%$ & 96 & $60.8 \%$ & 61 & $60.4 \%$ & 25 & $59.5 \%$ \\
\hline & Total & 97 & $100.0 \%$ & 143 & $100.0 \%$ & 158 & $100.0 \%$ & 101 & $100.0 \%$ & 42 & $100.0 \%$ \\
\hline \multirow{3}{*}{$\begin{array}{l}\text { 2-Prevent } \\
\text { colorectal } \\
\text { cancer }\end{array}$} & yes & 23 & $23.7 \%$ & 38 & $26.6 \%$ & 60 & $38.0 \%$ & 27 & $26.7 \%$ & 11 & $26.2 \%$ \\
\hline & no & 74 & $76.3 \%$ & 105 & $73.4 \%$ & 98 & $62.0 \%$ & 74 & $73.3 \%$ & 31 & $73.8 \%$ \\
\hline & Total & 97 & $100.0 \%$ & 143 & $100.0 \%$ & 158 & $100.0 \%$ & 101 & $100.0 \%$ & 42 & $100.0 \%$ \\
\hline \multirow{3}{*}{$\begin{array}{l}\text { 3-Prevent } \\
\text { ovarian cancer }\end{array}$} & yes & 9 & $9.3 \%$ & 20 & $14.0 \%$ & 32 & $20.3 \%$ & 8 & $7.9 \%$ & 8 & $19.0 \%$ \\
\hline & no & 88 & $90.7 \%$ & 123 & $86.0 \%$ & 126 & $79.7 \%$ & 93 & $92.1 \%$ & 34 & $81.0 \%$ \\
\hline & Total & 97 & $100.0 \%$ & 143 & $100.0 \%$ & 158 & $100.0 \%$ & 101 & $100.0 \%$ & 42 & $100.0 \%$ \\
\hline \multirow[t]{3}{*}{ 4-Lose weight } & yes & 23 & $23.7 \%$ & 45 & $31.5 \%$ & 41 & $25.9 \%$ & 29 & $28.7 \%$ & 9 & $21.4 \%$ \\
\hline & no & 74 & $76.3 \%$ & 98 & $68.5 \%$ & 117 & $74.1 \%$ & 72 & $71.3 \%$ & 33 & $78.6 \%$ \\
\hline & Total & 97 & $100.0 \%$ & 143 & $100.0 \%$ & 158 & $100.0 \%$ & 101 & $100.0 \%$ & 42 & $100.0 \%$ \\
\hline \multirow{3}{*}{$\begin{array}{l}\text { 5-Reduces risk } \\
\text { DM }\end{array}$} & yes & 33 & $34.0 \%$ & 49 & $34.3 \%$ & 58 & $36.7 \%$ & 31 & $30.7 \%$ & 8 & $19.0 \%$ \\
\hline & no & 64 & $66.0 \%$ & 94 & $65.7 \%$ & 100 & $63.3 \%$ & 70 & $69.3 \%$ & 34 & $81.0 \%$ \\
\hline & Total & 97 & $100.0 \%$ & 143 & $100.0 \%$ & 158 & $100.0 \%$ & 101 & $100.0 \%$ & 42 & $100.0 \%$ \\
\hline \multirow[t]{3}{*}{ 6-Oral health } & yes & 54 & $55.7 \%$ & 84 & $58.7 \%$ & 89 & $56.3 \%$ & 71 & $70.3 \%$ & 20 & $47.6 \%$ \\
\hline & no & 43 & $44.3 \%$ & 59 & $41.3 \%$ & 69 & $43.7 \%$ & 30 & $29.7 \%$ & 22 & $52.4 \%$ \\
\hline & Total & 97 & $100.0 \%$ & 143 & $100.0 \%$ & 158 & $100.0 \%$ & 101 & $100.0 \%$ & 42 & $100.0 \%$ \\
\hline
\end{tabular}

\section{Discussion}

Milk is main contributor of basic nutrients that are required for normal growth and development and prevent of many disease [22].

This study determined that there was significant number of students at ages between 21-23 years old involved in the study and also more female to male 1: 0.8 and this was the fact of students at medical faculties according the registry data by which female twice than male. Similar results have been found by other authors $[1,2]$.

According to the present study most of the students drink milk which represent by $82.6 \%$ and dairy products $97.2 \%$ by which one serving/ day $(P<0.05)$, drink whole fat milk $(P<0.05)$ and the consumption of milk habits revealed that significant numbers of students drink milk with tea $(P<0.05)$. The results of our study were inconsistent with Jafari $e t$ al, who concluded that $78.1 \%$ of the participants did not consume enough dairy products [8]. Maryam et al. also reported that the average milk consumption of students was as low as $100 \mathrm{~g}$, which was only one third of their recommended daily amount [1]. According to Rizzoli and et al adequate servings of dairy products were required to meet the recommended calcium intake for bone health and fitness [9]. Furthermore, a study conducted at Kafkas University to access the milk consumption patterns of students and the results concluded that only $33 \%$ of them consume milk while $67 \%$ of them did not consume milk throughout the study [10] Similar findings have been found and reported by national health and nutrition of America in which $39 \%$ of men and $43 \%$ of women consumed less and even less than single serving of milk and dairy products on the daily basis [10]. In fact that, decrement serving size of drink milk in our study could contribute to calcium and vitamin D deficiency and this due to the milk and dairy product serving found less than 
the recommended and it well known milk and dairy products are good sources of vitamin D and calcium [23, 24]. Milk has low amounts of iron, and its bioavailability is low. It is known that milk cannot meet iron requirements [24] .

For habits of drinking milk, there was a number of studies shown that students preferred drink milk with coffee [25]. But our result indicated that significant numbers of students which present by about $65 \%$ drink milk with tea.

Milk contains a number of vitamins, minerals and other nutrients and one of the best sources of protein [11-13]. The nutritional knowledge of milk and dairy products consumption were shown very good knowledge for numbers of questions listed in table 5. These knowledge were generally found among all students in medical faculties branches. There were also several studies reported similar results [26, 27]. This indicated our students have good idea for nutritional components of the milk.

Milk is regarded as a 'complete food' and the results of the present study showed that most of our students did not know about the importance of milk for our health and this could be related to milk and dairy products serving preferences as mentioned above and also could be due to other factors by which college and university life are considered 'busiest' due to the study burden and tough schedule [28] and also because of many other factors such as food preferences, expectations as well as changes in lifestyles and increased availability of fast foods were responsible towards the selection of foods among students. College life is considered as a period of students gain independence from their parents and this period is crucial in which turning point of food habits that have their implications through the adulthood. Sakamaki and et al, in China and reported that only $7 \%$ of the university students follow the concept of healthy eating while selecting food [13]. According to what have been mentioned above for poor knowledge of health benefit of dairy and dairy products there were a number of studies found similar results $[29,30]$.

Nutritional status of the students revealed that majority of the student fall under normal body weight BMI (18.5-24.9) and this probably explained by Forshee and et al decreased milk consumption was associated with change in the body weight and it was indirectly linked to wrong food choices and negative dietary patterns [14]. Although in current work as many as $82 \%$ of the students were drink milk so that the normal body weight found in this study was in parallel with high number of milk and dairy products consumer which prevent development of obesity. Furthermore studies by Maryam and et al, and Ozdogan and et al conducts on university students and their result were conclusive that the majorities of the students have normal body weight $[1,2]$. In addition, there was reported that a number of students have vitamin $D$ deficiency and low hemoglobin values and this in part due to low consumption of milk and dairy products and habit of milk drinking with tea could contribute to less iron absorption.

This study suggested that nutrition interventions were important in improving the diet quality by changing the nutrition knowledge as well as food habits of the target population. Furthermore, this study need further validated in large samples.

\section{Conclusion}

This study conducts on students from medical faculties braches and the result of this study revealed that students consumed one serving of milk on the daily basis , consumed whole fat milk and habit milk consumption significant was with tea. Although, vitamin D deficiency and low levels of hemoglobin were common. In regard milk knowledge, high nutritional knowledge scores have been revealed among students but poor knowledge were reported regarding health benefit of milk and dairy products consumption except for oral health. The result of nutritional status by BMI indicated majorities of students fall under normal BMI. This data suggested that high nutritional knowledge and poor health benefit knowledge need to be further investigated. Its highly recommended that students should be educated for the daily recommended intake of milk and dairy products in order to achieve good health and vitamin D status.

\section{Compliance with ethical standards}

\section{Acknowledgments}

We are grateful to all subjects who participated in the study.

\section{Disclosure of conflict of interest}

No conflict of interest. 


\section{Statement of informed consent}

All of the procedures involving human subjects were approved by local Medical Ethics committee. Written consent was obtained from the students before begun of the study.

\section{References}

[1] Maryam F, Chughtai A, Iqbal S, Tahir SK. Impact of nutrition education interventions on milk consumption among students (20-22 years). Life Science Journal of Pakistan. 2019; 1(1): 34-39.

[2] Yahya 0, Hülya Y, Ayse 00. Young adults' milk consumption habits and knowledge about milk. Studies on EthnoMedicine. 2017; 11(1): 106-113.

[3] Dietz WH, Gortmaker SL. Preventing obesity in children and adolescents. Annu Rev Public Health. 2001; 22: 33753.

[4] Dror DK. Dairy consumption and pre-school, school-age and adolescent obesity in developed countries: a systematic review and meta-analysis. Obes Rev. 2014; 15(6): 516-27.

[5] Huncharek M, Muscat J, Kupelnick B. Impact of dairy products and dietary calcium on bone-mineral content in children: results of a meta-analysis. Bone. 2008; 43(2): 312-21.

[6] Bonjour JP, Kraenzlin M, Levasseur R, Warren M, Whiting S. Dairy in adulthood: from foods to nutrient interactions on bone and skeletal muscle health. J Am Coll Nutr. 2013; 32(4): 251-63.

[7] Soedamah-Muthu SS, Ding EL, Al-Delaimy WK, Hu FB, Engberink MF, Willett WC. Milk and dairy consumption and incidence of cardiovascular diseases and all-cause mortality: dose-response meta-analysis of prospective cohort studies. Am J Clin Nutr. 2011; 93(1): 158-71.

[8] Jafari F, Beladian-Behbahan SE, Samadpour M. Application of the stages of change model to dairy consumption among students of Shahrekord University of Medical Sciences. Journal of Shahrekord University of Medical Science. 2014; 15(6): 65-74.

[9] Rizzoli R. Dairy products, yogurts, and bone health. Am J Clin Nutr. 2014; 99(5): 1256S-62S.

[10] Çetinkaya A. A survey of the consumption habits of milk and milk products among the students in Kafkas University, Ataturk University. J Vet Sci. 2010; 5(2): 73-84.

[11] Mozaffarian D. The great fat debate: Taking the focus off of saturated fat. J Am Diet Assoc. 2011; 111(5): 665-666.

[12] Claeys WL, Verraes C, Cardoen S, De Block J, Huyghebaert A. Consumption of raw or heated milk from different species: An evaluation of the nutritional and potential health benefits. Food Control. 2014; 42: $188-201$.

[13] Ruka S, Kenji T, Rie A. Nutritional knowledge, food habits and health attitude of Chinese university students -a cross sectional study. Nutr J.2005; 4(4): 15-20.

[14] Pei-Lin H. Factors influencing students' decisions to choose healthy or unhealthy snacks at the University of Newcastle, Australia. J Nusr R. 2004; 12(2): 83-91.

[15] de Goede J, Soedamah-Muthu SS, Pan A, Gijsbers L, Geleijnse JM. Dairy consumption and risk of stroke: a systematic review and updated dose response meta-analysis of prospective cohort studies. J Am Heart Assoc. 2016; 5(5): 123-132.

[16] Rice BH. Dairy and cardiovascular disease: a review of recent observational research. Curr Nutr Rep. 2014; 3: 130-8.

[17] Qin LQ, Xu JY, Han SF, Zhang ZL, Zhao YY, Szeto IM. Dairy consumption and risk of cardiovascular disease: an updated meta-analysis of prospective cohort studies. Asia Pac J Clin Nutr. 2015; 24(1): 90-100.

[18] Lampe JW. Dairy products and cancer. J Am Coll Nutr. 2011; 30(5 Suppl 1): 464S-70S.

[19] Miller GD, Jarvis JK, McBean LD. Hand book of Dairy foods and nutrition. 3rd ed. London: Taylor \& Frances Group. 2007.

[20] Abargouei AS, Janghorbani M, Salehi-Marzijarani M, Esmaillzadeh A. Effect of dairy consumption on weight and body composition in adults: a systematic review and metaanalysis of randomized controlled clinical trials. Int J Obes (Lond). 2012; 36(12): 1485-93. 
[21] Alwerfaly, M, Tarkhan A, Alzowi S, and Elmabsout AA. Ketogenic Diet Practices for Weight Management and Health Outcomes. International Journal of Applied Sciences: Current and Future Research Trends. 2021; 10(01): 16-30.

[22] Faghih A, Anoosheh M, Ahmadi F, Ghofranipoor F. The effect of boy students' participation on consumption of milk and dairy. Hormozgan Medical Journal. 2007; 10(4): 349-56.

[23] Hosseini, Z, Gharlipour G, Zabihollah MS, Sharifirad G, Mohammadbeigi AZ. Associated factors of milk consumption among students: Using Health Belief Model (HBM). 2017; 5: 4439-4448.

[24] Trave, T. Intake of milk and dairy products in a college population. Nutrición hospitalaria : organo oficial de la Sociedad Española de Nutrición Parenteral y Enteral. 2008; 23: 89-94.

[25] Hosseini Z, Gharlipour Z, Mohebi S, Sharifirad Gh, Mohammadbeigi A, Kazazloo Z. Associated Factors of Milk Consumption among Students: Using Health Belief Model (HBM). Int J Pediatr. 2017; 5(2): 4439-48.

[26] Mahon AK, Haas EJ. A mixed-methods approach to targeting college students' dairy behaviors. American Journal of Health Behavior. 2013; 37(5): 703-10.

[27] Lee, L, Jung I. A Study on Consumption Behavior of Milk and Dairy products in College Students. Journal of the Korean Society of Food Culture. 2002; 17: 123-134.

[28] Stearns SF, Rabinowitz AN. Understanding Milk Consumption Habits Among College Students in Order to Redesign Outreach. Journal of Extension. 2021; 59(2): 1-9.

[29] Rose AM, Williams RA, Rengers B, Kennel JA, Gunther C. Determining attitudinal and behavioral factors concerning milk and dairy intake and their association with calcium intake in college students. Nutrition Research and Practice. 2018; 12(2): 143-148.

[30] Kim SH, Kim WK, Kang MH. Relationships between milk consumption and academic performance, learning motivation and strategy, and personality in Korean adolescents. Nutr Res Pract. 2016; 10(2): 198-205. 